\title{
Automatic Recognition, Segmentation, and Sex Assignment of Nocturnal Asthmatic Coughs and Cough Epochs in Smartphone Audio Recordings: Observational Field Study
}

Filipe Barata ${ }^{1}$, MSc; Peter Tinschert ${ }^{2}$, MSc; Frank Rassouli ${ }^{3}, \mathrm{MD}, \mathrm{PhD}$; Claudia Steurer-Stey ${ }^{4,5}$, MD, PhD; Elgar Fleisch $^{1,2}$, PhD; Milo Alan Puhan ${ }^{4}$, MD, PhD; Martin Brutsche ${ }^{3}$, MD, PhD; David Kotz ${ }^{1,6,7}$, PhD; Tobias Kowatsch ${ }^{1,2}$, $\mathrm{PhD}$

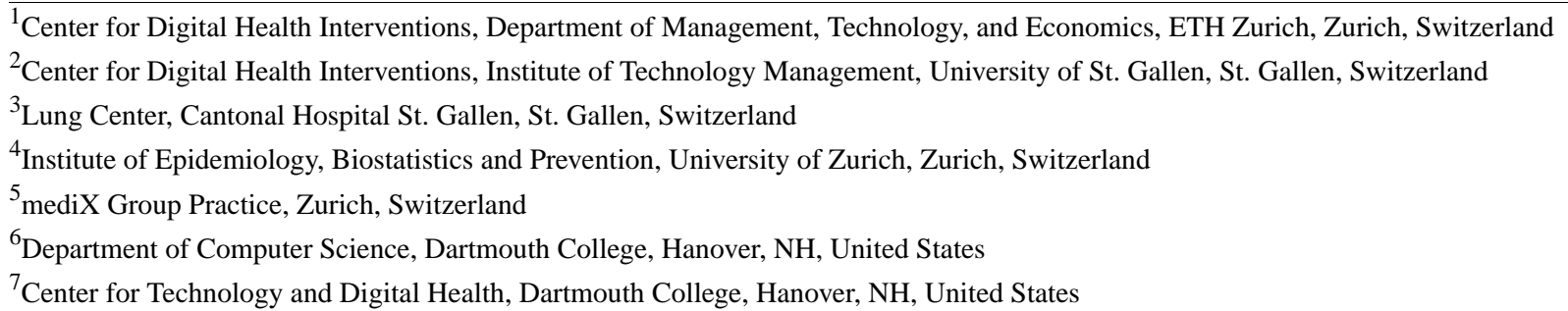

\section{Corresponding Author:}

Filipe Barata, MSc

Center for Digital Health Interventions

Department of Management, Technology, and Economics

ETH Zurich

Weinbergstrasse 56/57

Zurich, 8092

Switzerland

Phone: 41446323509

Email: fbarata@ethz.ch

\section{Abstract}

Background: Asthma is one of the most prevalent chronic respiratory diseases. Despite increased investment in treatment, little progress has been made in the early recognition and treatment of asthma exacerbations over the last decade. Nocturnal cough monitoring may provide an opportunity to identify patients at risk for imminent exacerbations. Recently developed approaches enable smartphone-based cough monitoring. These approaches, however, have not undergone longitudinal overnight testing nor have they been specifically evaluated in the context of asthma. Also, the problem of distinguishing partner coughs from patient coughs when two or more people are sleeping in the same room using contact-free audio recordings remains unsolved.

Objective: The objective of this study was to evaluate the automatic recognition and segmentation of nocturnal asthmatic coughs and cough epochs in smartphone-based audio recordings that were collected in the field. We also aimed to distinguish partner coughs from patient coughs in contact-free audio recordings by classifying coughs based on sex.

Methods: We used a convolutional neural network model that we had developed in previous work for automated cough recognition. We further used techniques (such as ensemble learning, minibatch balancing, and thresholding) to address the imbalance in the data set. We evaluated the classifier in a classification task and a segmentation task. The cough-recognition classifier served as the basis for the cough-segmentation classifier from continuous audio recordings. We compared automated cough and cough-epoch counts to human-annotated cough and cough-epoch counts. We employed Gaussian mixture models to build a classifier for cough and cough-epoch signals based on sex.

Results: We recorded audio data from 94 adults with asthma (overall: mean 43 years; SD 16 years; female: 54/94, 57\%; male 40/94, 43\%). Audio data were recorded by each participant in their everyday environment using a smartphone placed next to their bed; recordings were made over a period of 28 nights. Out of 704,697 sounds, we identified 30,304 sounds as coughs. A total of 26,166 coughs occurred without a 2-second pause between coughs, yielding 8238 cough epochs. The ensemble classifier performed well with a Matthews correlation coefficient of $92 \%$ in a pure classification task and achieved comparable cough counts to that of human annotators in the segmentation of coughing. The count difference between automated and human-annotated 
coughs was a mean $-0.1(95 \% \mathrm{CI}-12.11,11.91)$ coughs. The count difference between automated and human-annotated cough epochs was a mean 0.24 (95\% CI -3.67, 4.15) cough epochs. The Gaussian mixture model cough epoch-based sex classification performed best yielding an accuracy of $83 \%$.

Conclusions: Our study showed longitudinal nocturnal cough and cough-epoch recognition from nightly recorded smartphone-based audio from adults with asthma. The model distinguishes partner cough from patient cough in contact-free recordings by identifying cough and cough-epoch signals that correspond to the sex of the patient. This research represents a step towards enabling passive and scalable cough monitoring for adults with asthma.

(J Med Internet Res 2020;22(7):e18082) doi: 10.2196/18082

\section{KEYWORDS}

asthma; cough recognition; cough segmentation; sex assignment; deep learning; smartphone; mobile phone

\section{Introduction}

Asthma is one of the most prevalent chronic respiratory diseases [1]; it is estimated to afflict 339 million people worldwide [1]. Despite increased investment in treatment, hospital admissions and mortality rates have remained stable over the last decade [2]. This stagnation highlights the lack of scalable and easy-to-manage diagnostic tools for early recognition of exacerbations [3]. Traditional asthma self-management programs include written plans of action that cover how to recognize and respond to exacerbations [4]. These pen-and-paper approaches have shown improved health outcomes such as reduced hospital admissions, better lung function, fewer asthma symptoms, and less use of rescue medication [4-6]. The implementation of these programs, however, is low in clinical practice, with only $27 \%$ of adults with asthma receiving an asthma action plan [7]. Also, patient adherence to written action plans is poor and declines over time [8], and due to the reliance on subjective information in action plans, patients may perceive their symptoms poorly, and thus, underestimate the severity of the disease [9].

Disease control (also referred to as asthma control) is defined as the degree to which symptoms are controlled by treatment [10]. Exacerbations of asthma occur gradually over several days to weeks on a background of poor asthma control $[11,12]$. Although there is consensus on the importance of longitudinal and objective assessment of asthma control in the home environment, few tools enable objective measurement [3]. Current guidelines for the assessment of asthma control recommend the measurement of peak expiratory flow [13]. Changes in peak expiratory flow can occur up to 2 weeks before an exacerbation [14]. This parameter, however, is dependent on effort [3], and only 5\% of adults with asthma measure peak expiratory flow regularly [15]; therefore, it has limited utility. Nocturnal cough is a physiologic parameter which has shown promise for use in the assessment of asthma. Cough is a particularly burdensome asthma symptom [16] and was shown to be associated with asthma severity [17] and a worse prognosis [18]. Cough counts per night (ie, the number of coughs produced by one individual per night) have been associated with the level of asthma control [19]. Moreover, lower levels of asthma control have been correlated with poor quality of life [20] and are a predictor for cost-of-illness [21]. Coughing may also provide valuable information to predict the effects of asthma therapy early on [22]. Thus, nocturnal cough monitoring may play an important role in the prevention of exacerbations and in the personalization of treatment.

The monitoring of coughing by quantifying the number of coughs per unit of time has been attempted by researchers since the 1950s [23]. Although cough events may be counted manually from sound and video recordings, this process is extremely laborious. In consequence, many semi and fully automated cough monitoring systems have been in development since the 1950s. Among the more well-known systems are the Hull Automatic Cough Counter [24], the Leicester Cough Monitor [25], and LifeShirt [26]. The Hull Automatic Cough Counter is a software program written in MATLAB with the ability to differentiate cough from noncough sounds using Sony Walkman tape recordings. Leicester Cough Monitor is based on a free-field microphone necklace that records sound continuously onto a digital recorder. Recordings can then be uploaded onto a computer where an automated cough detection algorithm analyzes them. LifeShirt is an ambulatory cardiorespiratory monitoring system with a unidirectional contact microphone. Though these systems lacked scalability and cost-effectiveness, they showed feasibility with respect to automatic cough detection and counting from audio recordings.

Smartphones are now ubiquitous [27] and are equipped with sensors capable of many types of monitoring with clinically valuable accuracy [28]. Their widespread adoption in all age groups enables them to be used for measurements within different population samples [29]. In addition, they can be used to passively monitor the health status of patients without an additional task of activating a monitor. Recent advances have used only a smartphone and its built-in microphone for cough monitoring [30-32].

To develop an automatic cough monitoring system is challenging due to the rare occurrence of coughing in comparison to the occurrence of other sounds. This natural imbalance of cough and noncough sounds poses two problems. First, it demands high specificity from the cough monitoring system to avoid false alarms from other similar and more frequently occurring sounds. Second, existing classification methods tend to perform poorly on minority-class examples when the data set is highly imbalanced [33]. In addition, experts have suggested sensitivities greater than or equal to $90 \%$ as necessary for clinical use [34]. An even greater challenge is to collect sufficient data in the intended context and timeframe to allow realistic assessment of the monitoring system, particularly 
with respect to respiratory conditions. Some cough monitoring systems have been developed based on data collected under lab conditions by recording voluntary coughs [35-37]. While this may be a valid approach to compare the performance of different classifiers, it may not represent the soundscape of a real-use case. No cough monitoring systems exist that have undergone longitudinal overnight testing nor do any exist that have been specifically evaluated in the context of asthma. Also, depending on the intended mode of use, new challenges arise for cough monitoring - distinguishing partner coughs from patient coughs in a room with two or more people using contact-free recordings is a difficult task. To date, there are no standardized methods, and there are no sufficiently validated cough monitors for general use that are commercially available and clinically acceptable [23].

The study was designed to mimic a real-world use case; data were collected from a smartphone placed on the bedside table in the participant's bedroom. The aim was to build a cough classifier and to evaluate its performance on unseen data. Further, we aimed to use the classifier to segment and count cough events over the course of the night. Building upon previous work [32], we adopted a convolutional neural network architecture, which performed best in comparison to that of other machine learning approaches when using voluntary cough data from different smartphone recordings. We altered the learning part of the algorithm by combining three different techniques from literature to combat the high class imbalance encountered in this real-life data set-ensemble learning [38], minibatch balancing [39], and decision thresholding [40].

Though a smartphone-based nocturnal cough monitoring service may enable passive monitoring in theory, its utility for application in practice depends on whether coughs can be correctly assigned to individuals. Prior research has shown that humans are able to determine whether the source of a cough is male or female based on sound alone [41]. In addition, sex-based differences in signal properties have been measured in cough signals [42]. Epidemiological research suggests that less than $10 \%$ of the general population identify as homosexual [43]. Assuming that most people either sleep alone or share their bedroom with a partner, for the vast majority of patients, correctly classifying cough by sex-based properties of the cough signal could allow a cough monitoring system to disregard the coughs that are not from the individual of interest. Therefore, in addition to cough detection, this work examines to what extent coughs can be correctly be classified by sex. This research represents a step towards enabling passive scalable monitoring for people with asthma.

\section{Methods}

\section{Overview}

This study involved the collection of smartphone-recorded audio and daily questionnaire data, the definition and quantification of coughs within that data (data annotation and automated cough recognition and segmentation), sex-based classification, and model performance evaluation.

\section{Data Collection}

We used data collected in a multicenter, longitudinal observational study over a 29-day period (28 nights) [41]. On the first and last day, participants underwent medical examination by health professionals at the study centers. At the start of the study, participants were equipped with a smartphone (Samsung Galaxy A3 2017, SM-A320FL) on which Clara-the study's chat-based app-was installed. This app was a study-specific enhancement of the mobile app in the open-source MobileCoach behavioral intervention platform [44,45]. At night, the app recorded audio data using the smartphone's microphone. It also delivered daily questionnaires to the patients, asking them (among other things) whether the participant slept alone.

All participant data were collected by the physician (asthma evaluation data) or the nurse (lung function evaluations) in the study centers and were transferred to an electronic format and stored online on the study server. Nightly sensor data were stored locally on the smartphone. Data were backed up to external hard drives and secure online storage once the participant had completed the study and had returned the smartphone.

The study protocol was reviewed and approved by the Ethikkommission Ostschweiz, which is responsible for research on humans in Eastern Switzerland (Business Management System for Ethics Committees ID: 2017-01872).

\section{Cough Definition and Quantification}

With respect to cough monitoring systems, the definition of cough depends on the modality used for monitoring [23]. In this study, we aimed to recognize coughs from sound recordings which can be done in several ways [23]. We focused on two methods: (1) counting explosive cough sounds and (2) counting cough epochs (continuous coughing sounds without a 2 -second pause [23]). The latter is derived from the first metric by computing the duration between explosive cough sounds.

\section{Data Annotation}

Before annotation, silence was marked by applying a decibel filter by means of the Audacity software to the recordings. The Sound Finder filter marked sounds below $-26 \mathrm{~dB}$ as silence with the constraint that the minimum duration of silence between sounds was 1 second. These periods marked as silence served as visual aids for the remainder of the annotation process. Human annotators listened to the smartphone recordings and labeled the periods that were not marked as silence as a cough if an explosive cough sound was identified [23,41].

We used two approaches to verify the quality of the labeling. First, we instructed human annotators to label an acoustic event if they were unsure that it was a cough. If annotators were unsure, the event was discarded and was not considered in the analysis. The remainder of acoustic events were classified as noncoughs. Second, the interrater reliability for the annotators was determined using intraclass correlation. A zero-inflated generalized mixed-effects model with a Poisson response was used [46]. Additional details of the annotation method can be found in Multimedia Appendix 1. 


\section{Cough Recognition and Segmentation}

\section{Data Set Partitioning for Cough Recognition}

When developing neural networks, the split into sets for training, validation, and testing of the model is favored over other approaches that involve cross-validation because of the long training phases of the models; however, this comes at the risk of overfitting the model to the specific data set and a lack of generalizability to unseen samples. To mitigate these effects, we split our data into disjunct data sets which contained a different set of participants in the training, validation, or test sets. Furthermore, the large number of cough samples and participants in comparison to former studies [7,24,25,30,31] may help mitigate the risk of overfitting.

Training, validation, and test sets were created in the following way. From participants with complete data sets, roughly $20 \%$ were drawn at random; these nocturnal audio recordings constituted the testing corpus for our evaluations. From the remaining participants, roughly $15 \%$ were drawn at random to be included in the validation set. The remaining participants then comprised the training set. Thus, data were roughly split into a ratio of $65: 15: 20$. For model evaluation, the neural network was first trained on the training set. Hyperparameter tuning and model selection were performed on the validation set. Once the best performing parameters and model were selected, the final training was completed on the unified training and validation set. Model results were derived from the test set. The data split proportions were motivated by the fact that larger amounts of training data improve the performance of the classifier [47].

\section{Neural Network Architecture for Cough Recognition}

This work was built upon a convolutional neural network architecture for cough recognition that we introduced in previous work [32] which recognized coughs in Mel spectrograms. Mel-scaled spectrograms are visual representations of audio signals with respect to time and frequency. Signal frequency ranges are Mel-scaled to represent the human perception of sound. In conjunction with convolutional neural network architectures, they have been reported to perform better than other time-frequency representations [48]. The detailed calculation of the Mel spectrograms used can be found in Multimedia Appendix 1. We evaluated this approach against different approaches for smartphone-based cough recognition in previous work on voluntary coughs and found that it performed best [32]. Our approach produced stable results across recordings of five different devices with different hardware and service life duration. Moreover, it was designed to be lightweight and its deployment and energy-efficiency were tested on smartphones. Figure 1 depicts the architecture.

Figure 1. The architecture consists of 5 convolutional layers with alternating max-pooling layers followed by a global max-pooling layer. The annotation "16@80x122" refers to a feature map with dimensions (height $\mathrm{x}$ width) and 16 channels. The annotation "1x7 Convolution" refers to a convolutional filter with spatial dimensions (height $\mathrm{x}$ width).

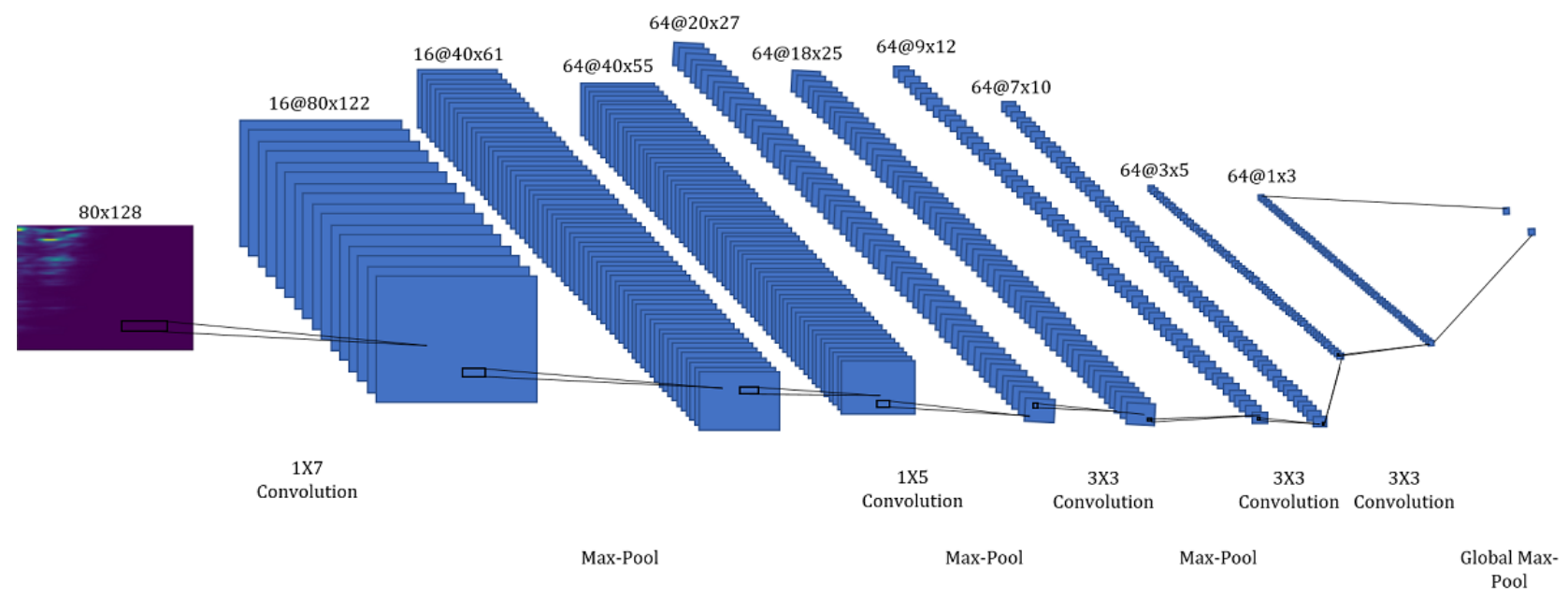

\section{Network Training for Cough Recognition}

\section{Overview}

A common problem in real data sets is that some classes have more samples than others. This class imbalance can have a considerable detrimental effect on convergence during the training phase and generalization of a model on the test set [40]. To counter the high imbalance in our data set, we employed 3 different techniques: ensemble learning, balanced minibatch learning, and decision thresholding. Our training approach can be summarized as follows. First, equally sized windows were extracted from the labeled acoustic cough and noncough events. From the resulting windows, we computed Mel spectrograms and employed data partitioning on the cough and noncough Mel spectrograms. Second, we created 5 folds out of the training data. Third, from each of those folds, a separate convolutional neural network model was trained using balanced minibatch training. These models had a probability score as output that indicated the likelihood of a cough. Finally, a threshold was determined to determine the averaged predicted probability of the trained models. The trained ensemble convolutional neural network classifier including thresholding is referred to as ensemble convolutional neural network throughout this paper.

\section{Window Extraction}

To train the classifier, nonoverlapping $650 \mathrm{~ms}$ windows from the noncough acoustic events were extracted. The duration of the window was based upon previous work. The same 
approximate duration has performed best in other cough monitoring approaches [49]. From the cough events, a single $650 \mathrm{~ms}$ window centered around the maximum amplitude was extracted since most cough events were shorter. Mel spectrograms were computed for these windows.

\section{Ensemble Learning}

In ensemble learning, combining individual models may help to improve generalization performance, if the individual models are dissimilar [50]. In previous work [32], we employed this approach by varying the subset of the devices from which single classifiers are trained, and thus created dissimilar classifiers. In this data set, we had a vast amount of recordings from one device model at our disposal. We, therefore, aimed to create dissimilar classifiers by training disjunct folds of the data instead. At the same time, by using disjunct folds, we reduced the training duration for a single classifier. Ensemble classifiers have also been applied successfully to other imbalanced data sets in prior work [38]. In this work, we subsampled the noncough class of the training data set into 5 participant-disjunct parts. For each of these subsamples and for the total sample of coughs in the training data, we trained a separate convolutional neural network classifier. In such a manner, we reduced the level of imbalance and still benefited from the increased prediction performance of an ensemble classifier. We limited our approach to 5 classifiers in order to limit computation, which increased linearly with the number of classifiers. To execute one of the convolutional neural network classifiers, 10.74 million floating-point operations are needed. The amount of computation may be of importance if the model is to be deployed on smartphones with limited computational resources. The output of each classifier is a probability score computed in the sigmoid neuron of the architecture.

\section{Balanced Minibatch Training}

Although the multilayer architecture of neural networks is essential, it is the back-propagation algorithm [51]that solves the optimization problem of minimizing the error between the samples and the predictions during the training phase using gradient descent. It computes the gradients of this error with respect to the neural network weights and propagates it back over the different layers defined by the architecture.

This process was done iteratively using the learning rule defined as follows:

$$
\theta^{\tau+1}:=\theta^{\tau}-\eta \sum_{i=1}^{n} \frac{\partial \mathrm{E}}{\partial \theta}
$$

where $n$ was the number of samples, $\theta^{\tau+1}$ was the new updated weight, $\theta^{\tau}$ was the current weight, $\eta$ was the learning rate, $E$ was the error, and $\partial$ was the differential operator. A more efficient technique for the evaluation of the learning rule is minibatch training [39] which introduces a trade-off when updating the weights. While using all training samples at once would have allowed for a precise update of the weights, it would also have taken longer and would have required excessive memory, and using a single instance to update the weights would have introduced noisy updates and would have been computationally inefficient. Minibatch training, however, splits the training data into small batches and computes weight updates from those batches. We employed balanced minibatch training, which also balanced the amount of majority and minority class instances per batch. Finally, we trained each of the convolutional neural network classifiers using balanced minibatch training and the Adam adaptive learning rate optimization algorithm [52]. This training approach relied on a number of hyperparameters, which were tuned and selected on the validation set, learning rate, batch size, and the number of iterations. Furthermore, weight initialization was accomplished using Xavier initialization [53] and dropout which is a regularization technique to prevent overfitting [54]. A dropout rate of $50 \%$ was used.

\section{Thresholding}

Thresholding adjusts the decision threshold of a classifier. It is typically applied in the test phase and involves changing the output class probabilities [40]. Since we used balanced minibatch training, in the training phase, we implicitly assumed that cough and noncough windows were each as likely to occur as the other. To account for the class imbalance and to find the best decision threshold, we employed a grid search on probabilities in the range of $[0.5,1)$ and tuned it on the validation set. The single classifier is one randomly selected classifier out of the 5 classifiers that constitute the ensemble classifier. We computed the threshold-based decision rule for the ensemble classifier as follows:

$$
\frac{1}{5} \sum_{i=1}^{5} Q_{i}\left(x \mid \lambda_{\text {cough }}\right) \begin{cases}<t & \text { noncough } \\ >t & \text { cough }\end{cases}
$$

where $Q_{i}\left(x \mid \lambda_{\text {cough }}\right)$ is the probability score of the classifier $i$ for the Mel spectrogram $x$ and the class cough $\lambda_{\text {cough }}$, and $t$ is the threshold.

\section{Cough and Cough-Epoch Segmentation}

In cough segmentation, the objective is to segment coughs from continuous audio recordings by employing a trained convolutional neural network ensemble classifier. We extracted $650 \mathrm{~ms}$ window with an overlap of $65 \mathrm{~ms}$. To discard silent windows, each of these windows passes through a decibel filter that removes windows with sounds below $-26 \mathrm{~dB}$ as in the annotation process. Subsequently, preprocessed into Mel spectrograms for each window were computed (as described in Multimedia Appendix 1). From these Mel spectrograms, cough probabilities were computed using the trained ensemble classifier. The continuous probabilities that were output were then transformed into segmentations by applying 3 postprocessing rules: (1) Only consecutive probabilities above the derived threshold were considered to be coughs, to reduce the number of false detections. (2) Single probabilities above the threshold were then considered, when the mean of the probability above the threshold and the following probability was above 0.9. (3) When more than eight consecutive detections occurred, they were recognized as two coughs. This was done to compensate for the limited resolution caused by the size of the overlap. These rules were derived by observation and yielded 
the best results on the validation set. Figure 2 illustrates the cough segmentation process. From the segmentation of cough, cough counts were computed. Cough epochs were recognized when two (or more) coughs occurred without a 2-second pause in between. An annotated cough epoch was considered to be correctly identified when at least one of the predicted coughs of the cough epoch corresponded with the annotated cough in the cough epoch.

Figure 2. The steps for the segmentation of coughs from continuous audio recordings (from top to bottom): First, the continuous extraction of overlapping windows from continuous audio recordings; second, the discarding of silent windows by applying a dB filter; third, the computation of Mel spectrograms; fourth, the computation of the prediction probability of cough by the convolutional neural network ensemble; last, the recognition of cough by applying the postprocessing rules. CNN: convolutional neural network.

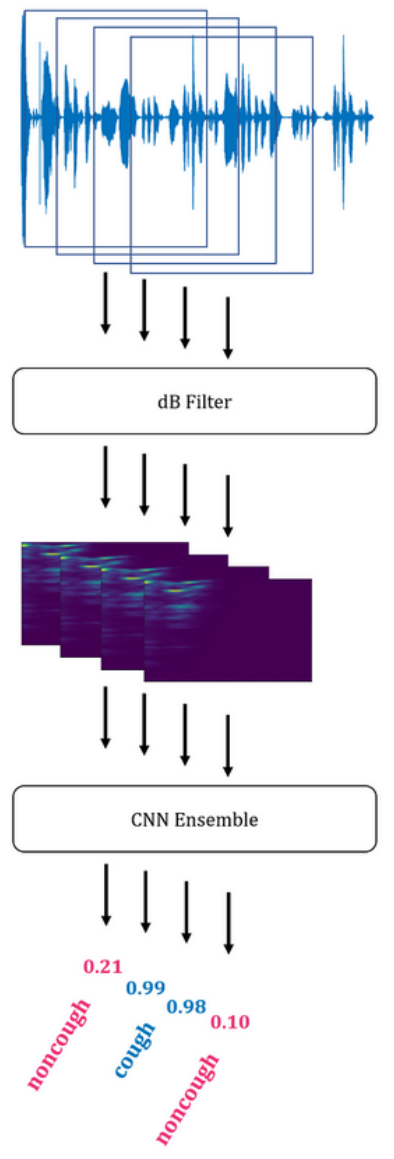

\section{Sex Classification}

\section{Data Set Partitioning}

For the data set partitioning for determining the source of each cough by sex, we used the complete data set and did not consider the partitioning used for cough recognition. The reason for this lay in the limited amount of data that fulfilled the filtering requirements for the analysis. Since the data collection study included couples or multiple people in one room, we filtered the annotated cough data based on the information collected daily regarding whether the participant slept alone or not. We then filtered the data of the corresponding nights to create a balanced data set of male and female coughs that included 19 female and 19 male participants. We conducted our analysis on both extracted cough and cough-epoch signals. In both cases, we partitioned the data set into a disjunct training set of 10 female and 9 male and a test set of 9 female and 10 male participants.

\section{Gaussian Mixture Models}

Gaussian mixture models in combination with Mel-frequency cepstral coefficients [55] are a known method for tackling several different recognition tasks in the audio domain, such as text-independent speaker recognition [56] or gender recognition from speech [57]. For the sex classification of cough signals, we used the $650 \mathrm{~ms}$ windows that were labeled as coughs and the resulting cough epochs to compute the Mel-frequency cepstral coefficients. In addition, their first time-derivative estimate and the first time-derivative estimate of the zero-crossing rate computed over the signal were used as features. The idea of training Gaussian mixture models was to approximate the probability distribution of a class by a linear combination of $K$ Gaussian distributions. The likelihood function of feature vector $X$ given class $\lambda$ can be described as follows:

$$
P(X \mid \lambda)=\sum_{k=1}^{K} \omega_{k} P_{k}\left(X \mid \mu_{k}, \Sigma_{k}\right)
$$

where $P_{k}\left(X \mid \mu_{k}, \Sigma_{k}\right)$ is the Gaussian distribution. The parameters mean $\mu_{k}$, covariance $\Sigma_{k}$, and weights $\omega$ of the distributions are determined during the training of the features $X$ of class $\lambda$. 
Considering the equal distribution of both sexes in the partitioned data set, a feature vector $x_{\mathrm{i}}$ of a cough or cough epoch can then be evaluated as follows:

$$
\log P\left(x_{i} \mid \lambda_{\text {female }}\right)-\log P\left(x_{i} \mid \lambda_{\text {male }}\right)\left\{\begin{array}{l}
>0 \text { female } \\
<0 \text { male }
\end{array}\right.
$$

Mel-frequency cepstral coefficients $(n=20)$ were computed with 256 samples between successive frames and a 4096-point fast Fourier transform. Analogously, the zero-crossing rate was computed over frames of 4096 samples with 256 samples between successive frames. These features were then vertically concatenated, which resulted in a matrix where the first dimension contained 41 entries. Feature selection and specific parameters were determined by employing 5-fold cross-validation on the training set. Hyperparameters of the Gaussian mixture models were also determined by employing 5-fold cross-validation on the training set which resulted in 30 Gaussian distributions each for female and male classes. Further hyperparameters were the number of initializations $(n=3)$, number of expectation-maximization iterations $(n=200)$, and the use of diagonal-type covariance.

\section{Performance Evaluation}

For the evaluation of the performance of the different models, we reported several metrics such as sensitivity (true positive rate), specificity (true negative rate), accuracy, Matthews correlation coefficients, predictive positive value, negative predictive value, receiver operating characteristic curve, precision-recall curve, and Bland-Altman plot. These metrics are commonly used in machine learning and research in the context of clinical decision-support systems. For the segmentation of cough and cough epoch, we reported the number of false positives, true positives, and false negatives per night. These metrics are defined in Multimedia Appendix 1.

\section{Results}

\section{Data}

\section{Participant Data}

A total of 94 participants (female: 54/94, 57\%; male: 40/94, $43 \%$ ) were recruited for the study. Ages of the participants ranged from 18 to 89 years with a mean of 43 (SD 16) years. Fifteen of the 94 participants were excluded from the analysis; 2 participants withdrew, 3 participants were not involved in the study procedures for more than 5 days, and 10 participants had more than 5 nights of missed audio recordings. Some of the missed audio recordings were due to technical difficulties (such as the app crashed) while some were participant-related (such as the participant's smartphone had been turned off).

\section{Data Set Partitioning}

\section{Cough Recognition}

Of the 79 participants whose data were included for analysis, 15 participants were initially drawn at random to be included in the test set. From the remaining 64 participants, 12 additional participants were drawn at random and included in the validation set. Data from the remaining 52 participants comprised the training set.

\section{Window Extraction}

Of a total of 704,697 acoustic events, 30,304 were clearly classified as coughing and $0.11 \%(767 / 704,697)$ were discarded. A total of 2,547,187 noncough and 30,304 cough Mel spectrograms were computed yielding a 0.015 class ratio.

\section{Thresholds}

Thresholds of 0.98 and 0.95 yielded best results in terms of Matthews correlation coefficient for the single and ensemble convolutional neural networks of $84 \%$ and $91 \%$ on the validation set, respectively.

\section{Annotator Intraclass Correlation}

Two of the annotators together accounted for $90.23 \%$ of all nights. These two annotators had an intraclass correlation of 95.79\% (mean absolute error: 0.44 coughs per night). We calculated the intraclass correlation based on 65 nights. The intraclass correlation was interpreted as excellent.

\section{Evaluation Cough Recognition Classifier Performance}

We evaluated the classifiers on the testing set, which consisted of 5489 cough and 541,972 noncough events. The test set represented the soundscape encountered in the bedrooms of 15 participants over the course of 28 nights. As shown in Table 1, the performance of the ensemble classifier was better than that of the single classifier. The difference was especially notable for true negative rate, negative predictive value, Matthews correlation coefficient values, and the area under the curve of the precision-recall curve. Both classifiers showed better performance for true positive rate compared to that for true negative rate which indicated a superior capability to recognize cough whenever a cough sound was presented compared to the capability to recognize noncough sounds. As a consequence, the false positive rate was higher than the false negative rate (Figure 3).

Table 1. Results of the convolutional neural network classifier for cough recognition.

\begin{tabular}{|c|c|c|c|c|c|c|}
\hline Model type & True positive rate, $\%$ & True negative rate, $\%$ & Accuracy, \% & $\begin{array}{l}\text { Matthews correla- } \\
\text { tion coefficient, } \%\end{array}$ & $\begin{array}{l}\text { Positive predictive } \\
\text { value, } \%\end{array}$ & $\begin{array}{l}\text { Negative predictive } \\
\text { value, } \%\end{array}$ \\
\hline Single & 99.9 & 87.5 & 99.7 & 87.2 & 99.9 & 87.1 \\
\hline Ensemble & 99.9 & 91.5 & 99.8 & 92.0 & 99.9 & 92.6 \\
\hline
\end{tabular}


Figure 3. Precision-recall curves with the corresponding area-under-the-curve values, for the single and ensemble convolutional neural network models for the recognition of coughing. The dashed line represents the curve for a random classifier showing the proportion of cough-class instances to the total amount of instances. AUC: area under the curve; CNN: convolutional neural network.

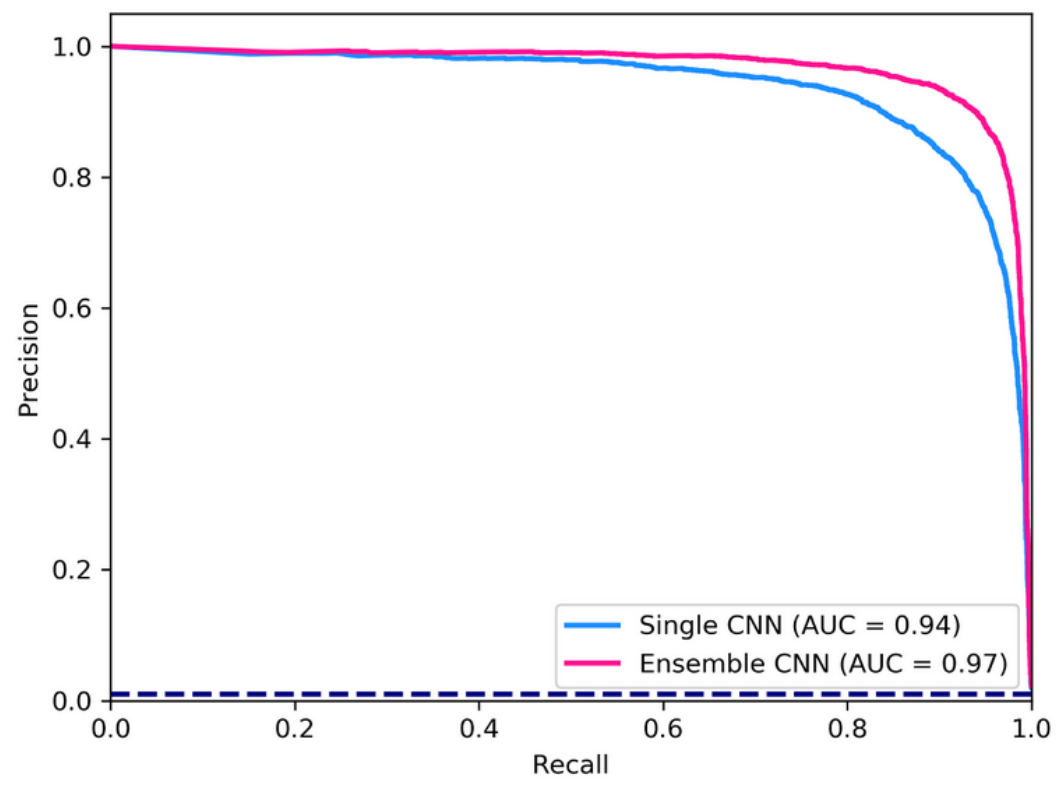

\section{Evaluation Cough and Cough-Epoch Segmentation}

The test set included 15 participants and 421 nights; human annotators counted as few as zero and as many as 368 coughs in one night. The mean count difference between automated and annotator coughs was $-0.1(95 \% \mathrm{CI}-12.11,11.91)$ coughs per night (Figure 4). Further, our classifier produced a mean of 1.76 false positives per night and 1.66 false negatives per night. In total, 241 nights were identified with a count difference of
0 coughs (Figure 5) and 5 nights had count differences greater than 20 coughs. Human annotators counted as few as zero and as many as 101 cough epochs in one night. The mean count difference between automated and observer cough epochs was 0.24 (95\% CI -3.67, 4.15) epochs per night (Figure 6). Our classifier produced a mean of 0.33 false positives per night and 0.57 false negatives per night. In total, 312 nights were identified with a count difference of 0 cough epochs (Figure 7) and 6 nights had count differences greater than 6 cough epochs.

Figure 4. Bland-Altman plot of the automated and annotator cough counts per night.

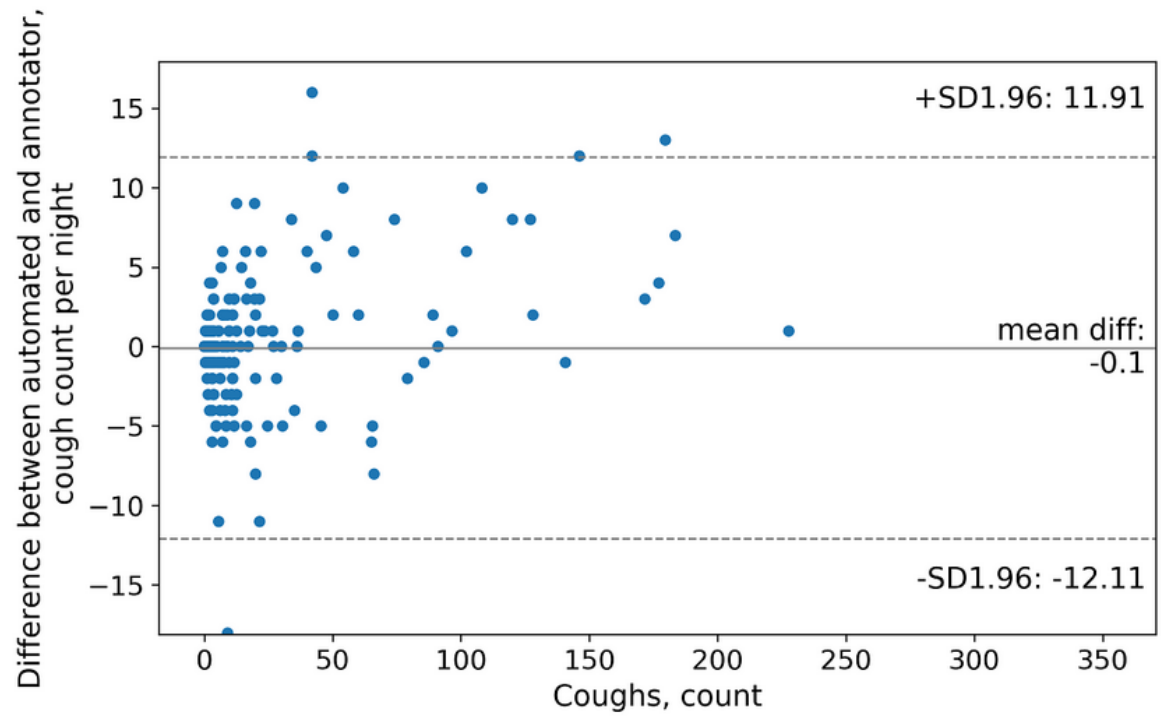


Figure 5. Histogram of the differences between automated and annotator cough counts per night.

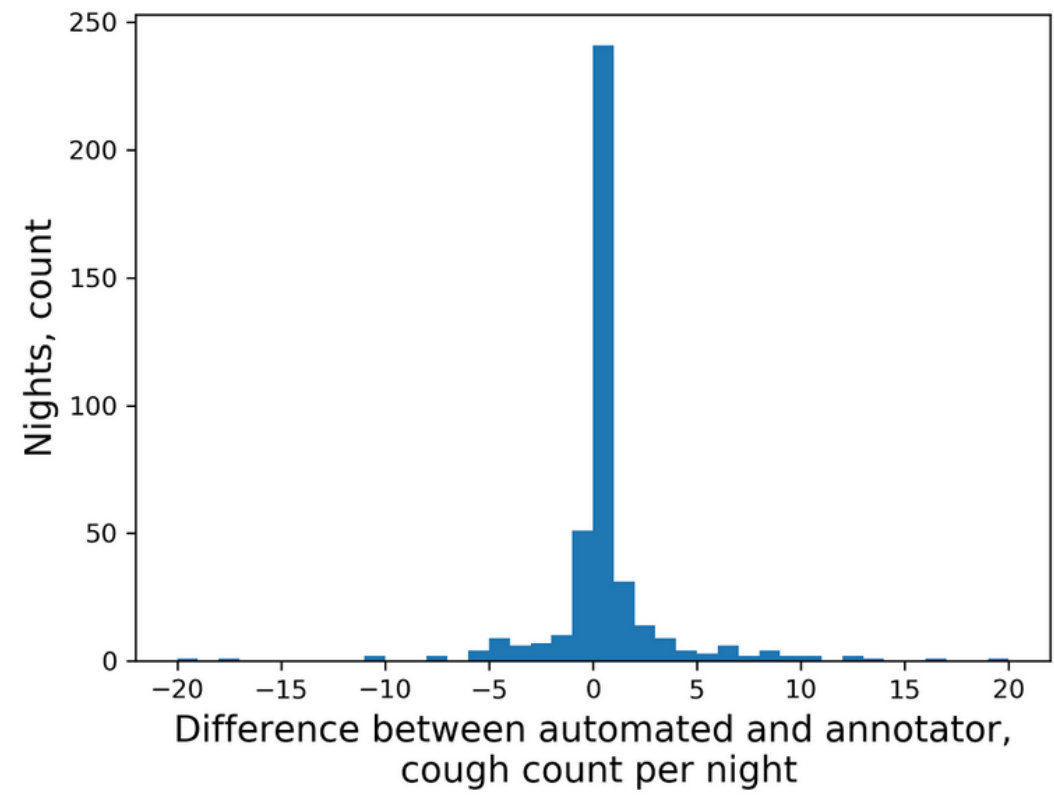

Figure 6. Bland-Altman plot of the automated and annotator cough-epoch counts per night.

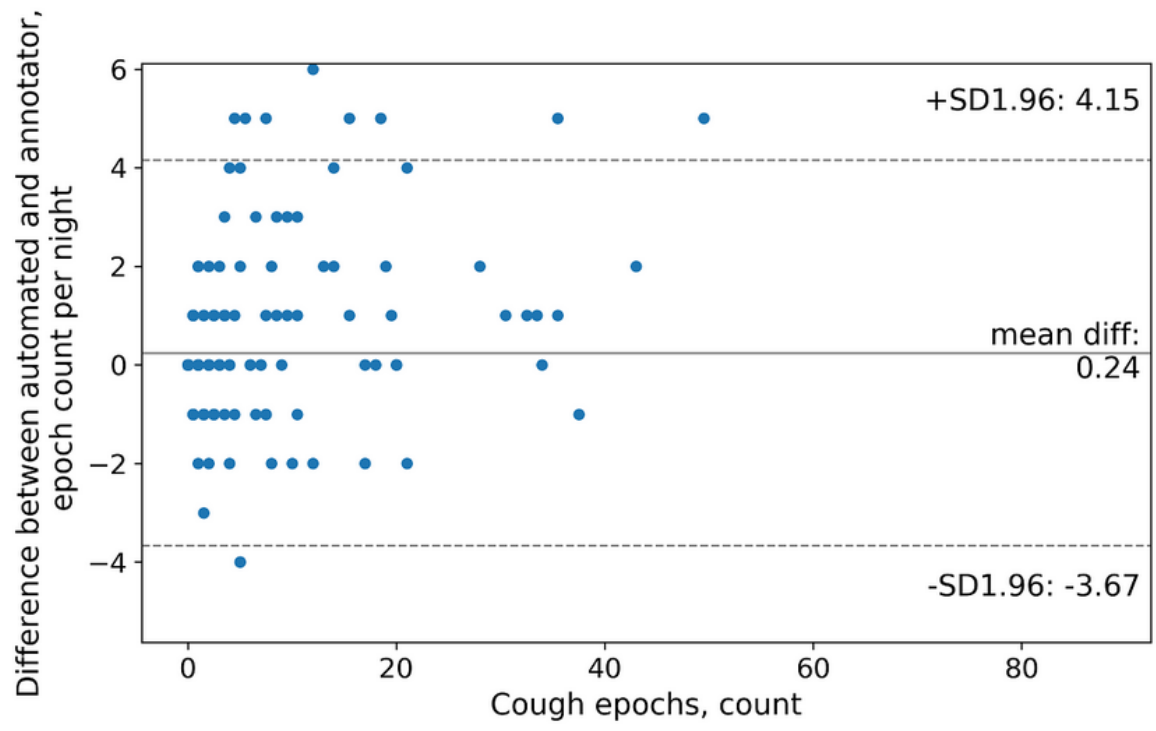


Figure 7. Histogram of the differences between automated and annotator cough-epoch counts per night.

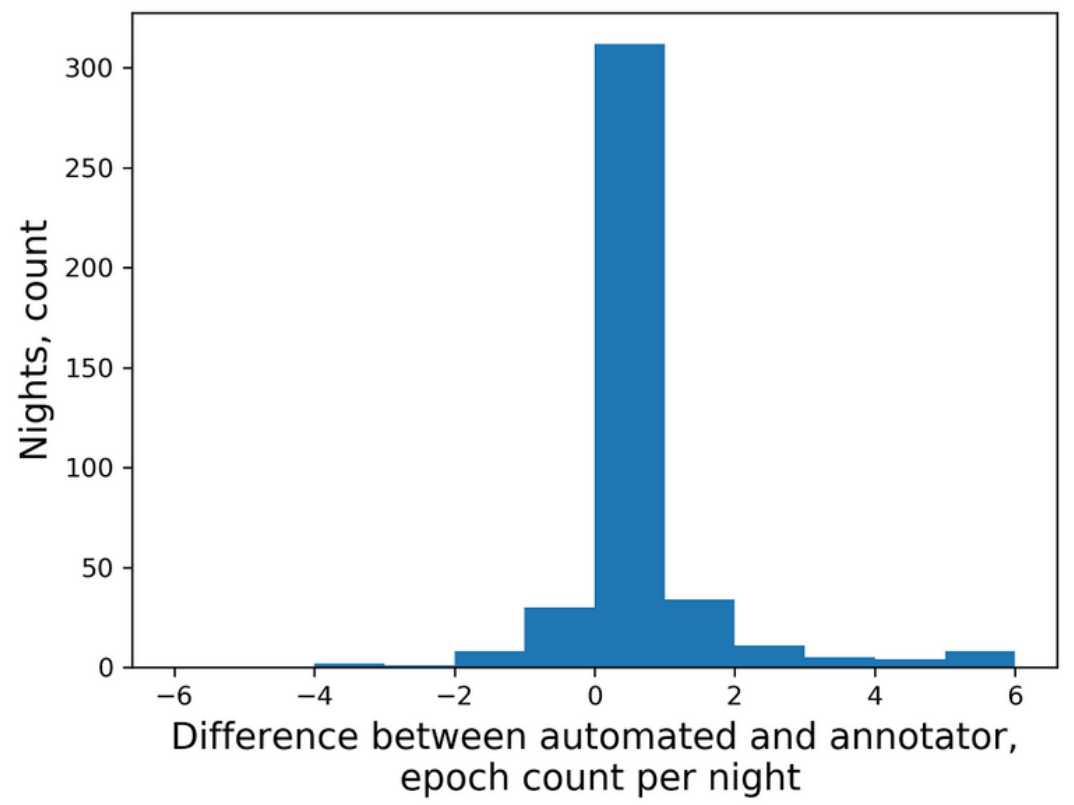

\section{Evaluation of Sex Classifier Performance}

Using the data set that included 19 female and 19 male participants which had been selected for a balanced set of male and female coughs, both extracted cough and cough-epoch signals were analyzed. The partitioning resulted in 1532 female and 1527 male coughs for training and 500 female and 498 male coughs for testing. In the case of cough epochs, this partitioning led to 366 female and 351 male cough epochs for training, and 194 male and 134 female cough epochs for testing.

As shown in Table 2 and Figure 8, the performance of the classifier that was based on cough-epoch signals outperformed the classifier based on cough signals. The difference was especially notable on true positive rate, accuracy, Matthews correlation coefficient, positive predictive values, negative predictive values, and the area under the curve of the receiver operating characteristic curve. Both classifiers showed better performance for true positive rate than for that of true negative rate, which indicated a superior capability to recognize female cough or cough-epoch signals whenever a female cough or cough-epoch signal presented itself, in comparison to the corresponding capability to recognize a male cough or cough-epoch signal.

Table 2. Gaussian mixture model results of sex recognition for coughs and cough epochs.

\begin{tabular}{lllllll}
\hline Model for & True positive rate, $\%$ & True negative rate, $\%$ & Accuracy, $\%$ & $\begin{array}{l}\text { Matthews correla- } \\
\text { tion coefficient, } \%\end{array}$ & $\begin{array}{l}\text { Positive predictive } \\
\text { value, } \%\end{array}$ & $\begin{array}{l}\text { Negative predictive } \\
\text { value, } \%\end{array}$ \\
\hline Cough & 81.0 & 71.8 & 74.8 & 49.6 & 57.8 & 88.8 \\
Cough epochs & 95.0 & 74.9 & 83.2 & 69.1 & 72.8 & 95.5 \\
\hline
\end{tabular}


Figure 8. Receiver operating characteristic curves with corresponding area-under-the-curve values for cough and cough epoch-based sex assignment. The dashed line represents the curve for a random classifier. AUC: area under the curve; ROC: receiver operating characteristic.

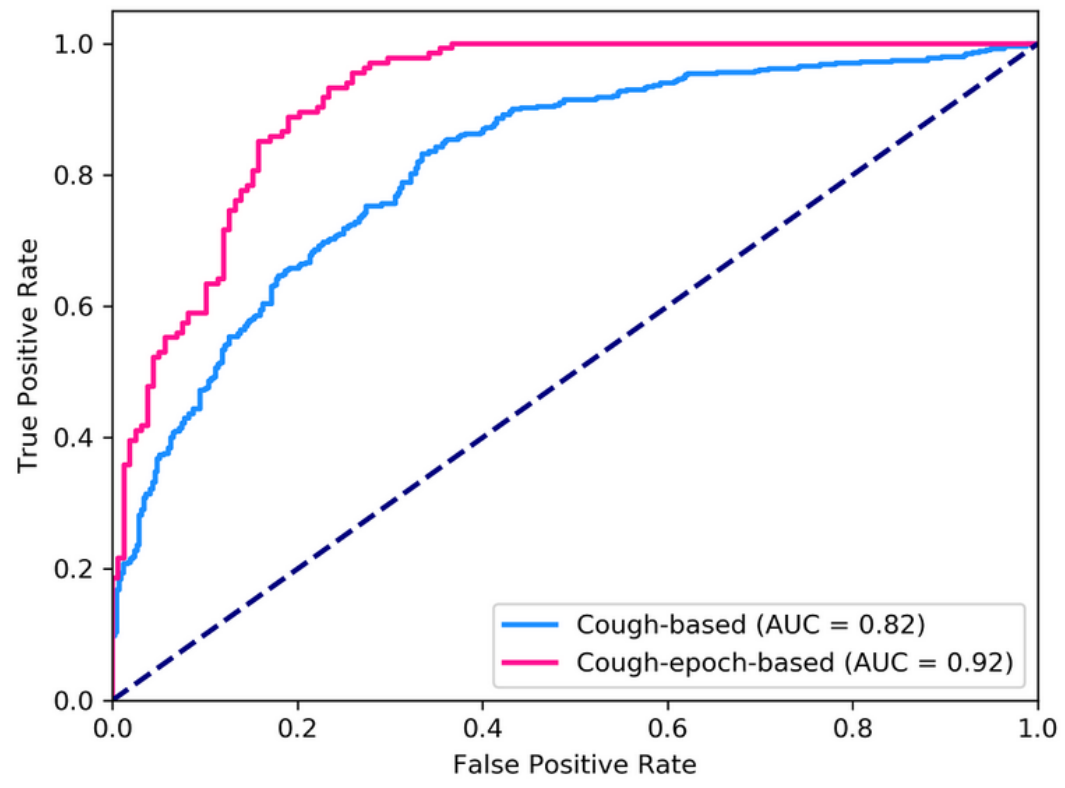

\section{Discussion}

\section{Principal Findings}

To the best knowledge of the authors, the data set in this paper is the largest, real-life cough data set with published recognition and segmentation results, not only for adults with asthma but across all respiratory conditions. Given the data set of continuous overnight recordings of 79 adults with asthma in different soundscapes (excluding dropouts), our results demonstrate that cough recognition from smartphone-based audio recordings is feasible. The ensemble classifier performed well with values greater than $90 \%$ across different metrics for the pure classification task and achieved comparable cough counts to that of human annotators in the segmentation of coughing from continuous overnight audio recordings. In specific cases (for example, the 6 nights with a difference in cough counts of 20 and above), a need for further development was demonstrated. We listened to the original recording of these cases and believe these failures were caused by strong background noise, peculiar chuckle and laughter sounds, and a specific type of music, among others. These sounds, however, strongly suggest that the participant was not asleep.

We also provided a first step towards distinguishing partner cough from patient cough by determining the source of cough signals classifying those that corresponded to sex of the patient as patient coughs. This can be applied to cough recordings from the bedrooms of opposite-sex couples, even when both are coughing. Our results further indicate that cough epoch-based sex classification has greater potential than that of cough-based sex classification. This may be explained by the fact that cough epochs are longer and may contain more periodic information, rather than the limited amount of periodic information contained in the short bursts of the explosive cough sound. Speech signals of a typical adult male contain a fundamental frequency from 85 to $180 \mathrm{~Hz}$ and those of a typical adult female from 165 to $255 \mathrm{~Hz}$ [58]. This discrepancy gives rise to sex classification from speech with greater than $90 \%$ of accuracy [59]. Finally, we also investigated the automatic segmentation of cough epochs from continuous audio recordings which yielded results that were comparable to those of annotated cough epochs. These results and the fact that $86 \%$ of the coughs in our study originated from cough epochs provided a foundation for cough epoch-based sex classification. Our classifier determined the source of cough epochs based on sex with $83 \%$ of accuracy.

\section{Comparison With Prior Work}

Cough-monitoring systems that are capable of detecting reflex coughs in audio recordings have been proposed in previous work $[24,25,30,60]$, and some of them have achieved sensitivity (true positive rate) and specificity (true negative rate) values greater than $90 \%$ [25,30,60]; however, these data sets contain coughs that have been recorded in various conditions and that have been applied in different contexts which makes a comparison with our work difficult. To our knowledge, no models or systems have been trained and evaluated on such an extensive asthmatic cough database; our data set contained 30,304 coughs. None of these systems underwent longitudinal evaluation (for more than one night). For instance, the Leicester Cough Monitor system was one of the few systems that was evaluated over a longer period of time; 6-hour and 24-hour cross-sectional recordings of patients with chronic cough [25]. Only a few approaches proposed modes of use that were comparable to our mode of use where the microphone was not attached to the patient $[24,31,60]$. Among those, only one involved a smartphone, where 1-hour recordings were collected for each participant in a laboratory setting [31,61]. None of these approaches addressed the problem of distinguishing the participant's cough from the coughs of other people in contact-free recordings.

\section{Limitations}

There were several limitations in our study regarding the generalization of our results. First, we only used data collected 
by one specific model of smartphone. It has previously been demonstrated [32] that noisy or low-quality recordings from a different device can have a detrimental effect on the performance of the classifiers. Second, the data set may limit the generalizability of our sex-classification results. For the analysis, we included data from different male and female participants, who slept alone but were recorded in different rooms, in contrast to a real scenario of a couple, where both are sleeping and coughing in the same room. Due to the amount and length of the recordings, the annotation process was extremely laborious. As a consequence, the majority of the recordings were only annotated by one annotator. This gives rise to the possibility that certain coughs were missed or wrongly annotated.

\section{Conclusions}

Our study proposed a combined approach to combat the detrimental effect of learning from highly imbalanced data sets by combining techniques such as ensemble learning, balanced minibatch training, and decision thresholding. We showed that automated methods can recognize nocturnal coughs and cough epochs in smartphone-based audio recordings. The model addressed distinguishing subject coughs from those of a bed partner in contact-free recordings by classifying cough and cough-epoch signals to the corresponding sex of the participant. This research enables smartphone-based cough monitoring of individuals and of couples of different sexes in their bedrooms. It represents a step towards passive, scalable cough monitoring for people with asthma, and thus contributes to the development of a scalable diagnostic tool for early recognition of exacerbations.

\section{Acknowledgments}

MB, MAP, PT, FB, CS-S, FR, and TK contributed to the study design. FR and CS-S were responsible for the study execution. PT and FB provided the technological support of the study execution. FB developed the study app and embedded the content created by PT and TK. The data annotation process was designed by PT and FB, and evaluated by PT. Data preprocessing, analysis, and machine learning were conducted by FB. Writing and editing of the manuscript was done by FB. Critical review and revision of the manuscript were done by DK, MB, MAP, TK, PT, CS-S, FR, and EF. FR was the principal investigator of the clinical study. This study was funded by CSS Insurance, Switzerland. The CSS insurance supported the recruitment of participants but had no role in study design, app design, data management plans, or in reviewing and approving the manuscript for publication. DK's participation in this research was funded by Dartmouth College and by the US National Institute of Drug Abuse through the Center for Technology and Behavioral Health at Dartmouth College. The views and conclusions contained in this document are those of the authors and do not necessarily represent the official policies, either expressed or implied, of the sponsors.

\section{Conflicts of Interest}

FB, PT, EF, and TK are affiliated with the Center for Digital Health Interventions, a joint initiative of the Department of Management, Technology, and Economics at Eidgenössische Technische Hochschule Zurich and the Institute of Technology Management at the University of St. Gallen, which is funded in part by the Swiss health insurer CSS. EF and TK are also cofounders of Pathmate Technologies, a university spin-off company that creates and delivers digital clinical pathways and has used the open-source MobileCoach platform for that purpose; however, Pathmate Technologies is not involved in the study app described in this paper.

EF and TK are also cofounders of Pathmate Technologies, a university spin-off company that creates and delivers digital clinical pathways and has used the open-source MobileCoach platform for that purpose; however, Pathmate Technologies is not involved in the study app described in this paper.

\section{Multimedia Appendix 1}

Additional information.

[DOCX File, 19 KB-Multimedia Appendix 1]

\section{References}

1. The Global Asthma Report 2018. Auckland, New Zealand: Global Asthma Network; 2018. URL: http://www. globalasthmareport.org/ [accessed 2020-06-03]

2. Pavord ID, Beasley R, Agusti A, Anderson GP, Bel E, Brusselle G, et al. After asthma: redefining airways diseases. Lancet 2018 Jan 27;391(10118):350-400. [doi: 10.1016/S0140-6736(17)30879-6] [Medline: 28911920]

3. Huffaker MF, Carchia M, Harris BU, Kethman WC, Murphy TE, Sakarovitch CCD, et al. Passive Nocturnal Physiologic Monitoring Enables Early Detection of Exacerbations in Children with Asthma. A Proof-of-Concept Study. Am J Respir Crit Care Med 2018 Aug 01;198(3):320-328 [FREE Full text] [doi: 10.1164/rccm.201712-26060C] [Medline: 29688023]

4. Gibson P, Powell H, Coughlan J, Wilson AJ, Abramson M, Haywood P, et al. Self-management education and regular practitioner review for adults with asthma. Cochrane Database Syst Rev 2003 Jul 22;3(1):CD001117. [doi: 10.1002/14651858.CD001117] [Medline: $\underline{\text { 12535399] }}$ 
5. Gibson PG, Powell H. Written action plans for asthma: an evidence-based review of the key components. Thorax 2004 Feb 01;59(2):94-99 [FREE Full text] [doi: 10.1136/thorax.2003.011858] [Medline: 14760143]

6. Adams R, Smith B, Ruffin R. Factors associated with hospital admissions and repeat emergency department visits for adults with asthma. Thorax 2000 Jul;55(7):566-573 [FREE Full text] [doi: 10.1136/thorax.55.7.566] [Medline: 10856316]

7. Honkoop PJ, Taylor DR, Smith AD, Snoeck-Stroband JB, Sont JK. Early detection of asthma exacerbations by using action points in self-management plans. Eur Respir J 2013 Jan;41(1):53-59 [FREE Full text] [doi: 10.1183/09031936.00205911] [Medline: 22653768]

8. Kaya Z, Erkan F, Ozkan M, Ozkan S, Kocaman N, Ertekin BA, et al. Self-management plans for asthma control and predictors of patient compliance. J Asthma 2009 Apr;46(3):270-275. [doi: 10.1080/02770900802647565] [Medline: 19373635]

9. Harkins MS, Fiato KL, Iwamoto GK. Exhaled nitric oxide predicts asthma exacerbation. J Asthma 2004 Jun;41(4):471-476. [doi: 10.1081/jas-120033990] [Medline: 15281333]

10. Schatz M, Sorkness CA, Li JT, Marcus P, Murray JJ, Nathan RA, et al. Asthma Control Test: reliability, validity, and responsiveness in patients not previously followed by asthma specialists. J Allergy Clin Immunol 2006 Mar;117(3):549-556. [doi: 10.1016/j.jaci.2006.01.011] [Medline: 16522452]

11. Chan-Yeung M, Chang JH, Manfreda J, Ferguson A, Becker A. Changes in peak flow, symptom score, and the use of medications during acute exacerbations of asthma. Am J Respir Crit Care Med 1996 Oct;154(4 Pt 1):889-893. [doi:

10.1164/ajrccm.154.4.8887581] [Medline: $\underline{8887581]}$

12. Turner MO, Noertjojo K, Vedal S, Bai T, Crump S, Fitzgerald JM. Risk factors for near-fatal asthma. A case-control study in hospitalized patients with asthma. Am J Respir Crit Care Med 1998 Jun;157(6 Pt 1):1804-1809. [doi: 10.1164/ajrccm.157.6.9708092] [Medline: 9620909]

13. Global Strategy for Asthma Management and Prevention, 2019. Global Initiative for Asthma. URL: https://ginasthma.org/ [accessed 2020-06-03]

14. Tattersfield AE, Postma DS, Barnes PJ, Svensson K, Bauer CA, O'Byrne PM, et al. Exacerbations of asthma: a descriptive study of 425 severe exacerbations. The FACET International Study Group. Am J Respir Crit Care Med 1999 Aug;160(2):594-599. [doi: 10.1164/ajrccm.160.2.9811100] [Medline: 10430734]

15. Murphy KR, Meltzer EO, Blaiss MS, Nathan RA, Stoloff SW, Doherty DE. Asthma management and control in the United States: results of the 2009 Asthma Insight and Management survey. Allergy Asthma Proc 2012;33(1):54-64. [doi: 10.2500/aap.2011.32.3518] [Medline: 22309716]

16. Osman L, McKenzie L, Cairns J, Friend J, Godden D, Legge J, et al. Patient weighting of importance of asthma symptoms. Thorax 2001 Feb;56(2):138-142 [FREE Full text] [doi: 10.1136/thorax.56.2.138] [Medline: 11209103]

17. de Marco R, Marcon A, Jarvis D, Accordini S, Almar E, Bugiani M, European Community Respiratory Health Survey Therapy Group. Prognostic factors of asthma severity: a 9-year international prospective cohort study. J Allergy Clin Immunol 2006 Jun;117(6):1249-1256. [doi: 10.1016/j.jaci.2006.03.019] [Medline: 16750983]

18. Thomson NC, Chaudhuri R, Messow CM, Spears M, MacNee W, Connell M, et al. Chronic cough and sputum production are associated with worse clinical outcomes in stable asthma. Respir Med 2013 Oct;107(10):1501-1508 [FREE Full text] [doi: 10.1016/j.rmed.2013.07.017] [Medline: 23927851]

19. Marsden PA, Satia I, Ibrahim B, Woodcock A, Yates L, Donnelly I, et al. Objective Cough Frequency, Airway Inflammation, and Disease Control in Asthma. Chest 2016 Jun;149(6):1460-1466. [doi: 10.1016/j.chest.2016.02.676] [Medline: 26973014]

20. Vollmer WM, Markson LE, O'Connor E, Sanocki LL, Fitterman L, Berger M, et al. Association of asthma control with health care utilization and quality of life. Am J Respir Crit Care Med 1999 Nov;160(5 Pt 1):1647-1652. [doi: 10.1164/ajrccm.160.5.9902098] [Medline: 10556135]

21. Accordini S, Corsico AG, Braggion M, Gerbase MW, Gislason D, Gulsvik A, et al. The cost of persistent asthma in Europe: an international population-based study in adults. Int Arch Allergy Immunol 2013;160(1):93-101. [doi: 10.1159/000338998] [Medline: 22948386]

22. Fukuhara A, Saito J, Birring SS, Sato S, Uematsu M, Suzuki Y, et al. Clinical Characteristics of Cough Frequency Patterns in Patients with and without Asthma. J Allergy Clin Immunol Pract 2020 Feb;8(2):654-661. [doi: 10.1016/j.jaip.2019.08.053] [Medline: 31541769 ]

23. Morice A, Fontana G, Belvisi M, Birring S, Chung K, Dicpinigaitis ROTAOC, European Respiratory Society (ERS). ERS guidelines on the assessment of cough. Eur Respir J 2007 Jun;29(6):1256-1276 [FREE Full text] [doi: 10.1183/09031936.00101006] [Medline: 17540788]

24. Barry SJ, Dane AD, Morice AH, Walmsley AD. The automatic recognition and counting of cough. Cough 2006 Sep 28;2:8 [FREE Full text] [doi: 10.1186/1745-9974-2-8] [Medline: 17007636]

25. Birring S, Fleming T, Matos S, Raj A, Evans D, Pavord I. The Leicester Cough Monitor: preliminary validation of an automated cough detection system in chronic cough. Eur Respir J 2008 May;31(5):1013-1018 [FREE Full text] [doi: 10.1183/09031936.00057407] [Medline: 18184683 ]

26. Wilhelm FH, Roth WT, Sackner MA. The lifeShirt. An advanced system for ambulatory measurement of respiratory and cardiac function. Behav Modif 2003 Oct;27(5):671-691. [doi: 10.1177/0145445503256321] [Medline: 14531161] 
27. Fox S, Duggan M. Tracking for health. Pew Research Center's Internet \& American Life Project. 2013. URL: https://www. pewresearch.org/internet/wp-content/uploads/sites/9/media/Files/Reports/2013/PIP TrackingforHealth-with-appendix.pdf [accessed 2020-06-03]

28. Juen J, Cheng Q, Schatz B. A natural walking monitor for pulmonary patients using mobile phones. IEEE J Biomed Health Inform 2015 Jul;19(4):1399-1405. [doi: 10.1109/JBHI.2015.2427511] [Medline: 25935052]

29. R Schatz B. Population measurement for health systems. Digit Med 2018;1:20174.

30. Larson E, Lee T, Liu S, Rosenfeld M, Patel S. Accurate and privacy preserving cough sensing using a low-cost microphone. 2011 Presented at: Proceedings of the 13th international conference on Ubiquitous computing; 2011; Beijing, China p. 375-384. [doi: 10.1145/2030112.2030163]

31. Monge-Alvarez J, Hoyos-Barcelo C, Lesso P, Casaseca-de-la-Higuera P. Robust Detection of Audio-Cough Events Using Local Hu Moments. IEEE J Biomed Health Inform 2019 Jan;23(1):184-196. [doi: 10.1109/JBHI.2018.2800741] [Medline: 29994432]

32. Barata F, Kipfer K, Weber M, Tinschert P, Fleisch E, Kowatsch T. Towards Device-Agnostic Mobile Cough Detection with Convolutional Neural Networks. 2019 Presented at: 7th IEEE International Conference on Healthcare Informatics (ICHI); 2019; Xi'an, China p. 1-11. [doi: 10.1109/ICHI.2019.8904554]

33. Rahman MM, Davis D. Addressing the Class Imbalance Problem in Medical Datasets. IJMLC 2013;3(2):224-228. [doi: 10.7763/IJMLC.2013.V3.307]

34. Smith J, Woodcock A. New developments in the objective assessment of cough. Lung 2008;186 Suppl 1:S48-S54. [doi: 10.1007/s00408-007-9059-1] [Medline: 18066694$]$

35. Vizel E, Yigla M, Goryachev Y, Dekel E, Felis V, Levi H, et al. Validation of an ambulatory cough detection and counting application using voluntary cough under different conditions. Cough 2010 May 27;6:3 [FREE Full text] [doi: 10.1186/1745-9974-6-3] [Medline: 20504377]

36. Drugman T, Urbain J, Dutoit T. Assessment of audio features for automatic cough detection. 2011 Presented at: 19th European Signal Processing Conference; 2011; Barcelona p. 1289-1293.

37. Amoh J, Odame K. Deep Neural Networks for Identifying Cough Sounds. IEEE Trans Biomed Circuits Syst 2016 Oct;10(5):1003-1011. [doi: 10.1109/TBCAS.2016.2598794] [Medline: 27654978]

38. Feng W, Huang W, Ren J. Class Imbalance Ensemble Learning Based on the Margin Theory. Applied Sciences 2018 May 18;8(5):815. [doi: 10.3390/app8050815]

39. Li M, Zhang T, Chen Y, Smola A. Efficient mini-batch training for stochastic optimization. 2014 Presented at: Proceedings of the 20th ACM SIGKDD international conference on Knowledge discovery and data mining; 2014; New York, USA p. 661-670. [doi: 10.1145/2623330.2623612]

40. Buda M, Maki A, Mazurowski MA. A systematic study of the class imbalance problem in convolutional neural networks. Neural Netw 2018 Oct;106:249-259. [doi: 10.1016/j.neunet.2018.07.011] [Medline: 30092410]

41. Tinschert P, Rassouli F, Barata F, Steurer-Stey C, Fleisch E, Puhan MA, et al. Prevalence of nocturnal cough in asthma and its potential as a marker for asthma control (MAC) in combination with sleep quality: protocol of a smartphone-based, multicentre, longitudinal observational study with two stages. BMJ Open 2019 Jan 07;9(1):e026323 [FREE Full text] [doi: 10.1136/bmjopen-2018-026323] [Medline: 30617104]

42. Rosenberry K, Goldsmith W, Reynolds J, McKinney W, Frazer D. Gender differences in voluntary cough sound spectra demonstrated by an inverse power law analysis. 2002 Presented at: Proceedings of the Second Joint 24th Annual Conference and the Annual Fall Meeting of the Biomedical Engineering Society; 2002; Houston, USA p. 222-223. [doi:

10.1109/iembs.2002.1134463]

43. Diamond M. Homosexuality and bisexuality in different populations. Arch Sex Behav 1993 Aug;22(4):291-310. [doi: 10.1007/BF01542119] [Medline: 8368913]

44. Filler A, Kowatsch T, Haug S, Wahle F, Staake T, Fleisch E. MobileCoach: A novel open source platform for the design of evidence-based, scalable and low-cost behavioral health interventions: Overview and preliminary evaluation in the public health context. 2015 Presented at: Wireless Telecommunications Symposium (WTS); 2015; New York, USA. [doi: 10.1109/WTS.2015.7117255]

45. Kowatsch T, Volland D, Shih I, Rüegger D, Künzler F, Barata F. Design and evaluation of a mobile chat app for the open source behavioral health intervention platform MobileCoach. 2017 Presented at: International Conference on Design Science Research in Information System and Technology; 2017; Karlsruhe, Germany p. 485-489. [doi:

10.1007/978-3-319-59144-5 36]

46. Koo TK, Li MY. A Guideline of Selecting and Reporting Intraclass Correlation Coefficients for Reliability Research. J Chiropr Med 2016 Jun;15(2):155-163 [FREE Full text] [doi: 10.1016/j.jcm.2016.02.012] [Medline: 27330520]

47. Banko M, Brill E. Scaling to very very large corpora for natural language disambiguation. 2001 Presented at: Proceedings of the 39th annual meeting on association for computational linguistics; 2001; Toulouse, France p. 26-33. [doi: $\underline{10.3115 / 1073012.1073017]}$

48. Huzaifah M. Comparison of time-frequency representations for environmental sound classification using convolutional neural networks. arXiv preprint arXiv. 2017. URL: https://arxiv.org/abs/1706.07156 [accessed 2020-06-03] 
49. Miranda I, Diacon A, Niesler T. A Comparative Study of Features for Acoustic Cough Detection Using Deep Architectures. In: Conf Proc IEEE Eng Med Biol Soc. 2019 Jul Presented at: 41st Annual International Conference of the IEEE Engineering in Medicine and Biology Society (EMBC); 2019; Berlin, Germany p. 2601-2605. [doi: 10.1109/EMBC.2019.8856412]

50. Zhou Z. Ensemble methods: foundations and algorithms. Boca Raton, Florida, USA: CRC press; 2012.

51. Rumelhart D, Hinton G, Williams R. Learning representations by back-propagating errors. Nature 1986 Oct;323(6088):533-536. [doi: 10.1038/323533a0]

52. Kingma D, Ba J. Adam: A method for stochastic optimization. 2015 Presented at: 3rd International Conference for Learning Representations; May 7-9, 2015; San Diego USA.

53. Glorot X, Bengio Y. Understanding the difficulty of training deep feedforward neural networks. 2010 Presented at: Proceedings of the thirteenth international conference on artificial intelligence and statistics; 13-15 May 2010; Chia Laguna Resort, Sardinia, Italy p. 249-256.

54. Hinton G, Srivastava N, Krizhevsky A, Sutskever I, Salakhutdinov R. Improving neural networks by preventing co-adaptation of feature detectors. arXiv preprint arXiv. 2012. URL: https://arxiv.org/abs/1207.0580 [accessed 2020-06-03]

55. Davis S, Mermelstein P. Comparison of parametric representations for monosyllabic word recognition in continuously spoken sentences. IEEE Trans. Acoust., Speech, Signal Process 1980 Aug;28(4):357-366. [doi: 10.1109/TASSP.1980.1163420]

56. Reynolds DA, Rose RC. Robust text-independent speaker identification using Gaussian mixture speaker models. IEEE Trans. Speech Audio Process 1995;3(1):72-83. [doi: 10.1109/89.365379]

57. Metze F, Ajmera J, Englert R, Bub U, Burkhardt F, Stegmann J. Comparison of four approaches to age and gender recognition for telephone applications. 2007 Presented at: 2007 IEEE International Conference on Acoustics, Speech and Signal Processing-ICASSP '07; 15-20 April 2007; Honolulu, USA. [doi: 10.1109/ICASSP.2007.367263]

58. Titze IR, Martin DW. Principles of voice production. The Journal of the Acoustical Society of America 1998 Sep;104(3):1148-1148. [doi: 10.1121/1.424266]

59. Childers DG, Wu K. Gender recognition from speech. Part II: Fine analysis. J Acoust Soc Am 1991 Oct;90(4 Pt 1):1841-1856. [doi: 10.1121/1.401664] [Medline: 1755877$]$

60. Amrulloh YA, Abeyratne UR, Swarnkar V, Triasih R, Setyati A. Automatic cough segmentation from non-contact sound recordings in pediatric wards. Biomedical Signal Processing and Control 2015 Aug;21:126-136. [doi: 10.1016/j.bspc.2015.05.001]

61. Monge PR, Contractor NS. Theories of Communication Networks. New York, USA: Oxford University Press; 2003.

Edited by G Eysenbach; submitted 03.02.20; peer-reviewed by L Zhang, G Lim; comments to author 03.03.20; revised version received 27.04.20; accepted 30.04.20; published 14.07.20

Please cite as:

Barata F, Tinschert P, Rassouli F, Steurer-Stey C, Fleisch E, Puhan MA, Brutsche M, Kotz D, Kowatsch T

Automatic Recognition, Segmentation, and Sex Assignment of Nocturnal Asthmatic Coughs and Cough Epochs in Smartphone Audio Recordings: Observational Field Study

J Med Internet Res 2020;22(7):e18082

URL: https://www.jmir.org/2020/7/e18082

doi: $\underline{10.2196 / 18082}$

PMID: $\underline{32459641}$

CFilipe Barata, Peter Tinschert, Frank Rassouli, Claudia Steurer-Stey, Elgar Fleisch, Milo Alan Puhan, Martin Brutsche, David Kotz, Tobias Kowatsch. Originally published in the Journal of Medical Internet Research (http://www.jmir.org), 14.07.2020. This is an open-access article distributed under the terms of the Creative Commons Attribution License (https://creativecommons.org/licenses/by/4.0/), which permits unrestricted use, distribution, and reproduction in any medium, provided the original work, first published in the Journal of Medical Internet Research, is properly cited. The complete bibliographic information, a link to the original publication on http://www.jmir.org/, as well as this copyright and license information must be included. 